\title{
ABORDAGEM TERAPÊUTICA NO PROCESSO DE ESVAZIAMENTO UTERINO
}

\author{
THERAPEUTIC APPROACH IN THE UTERINE \\ EVACUATION PROCESS
}

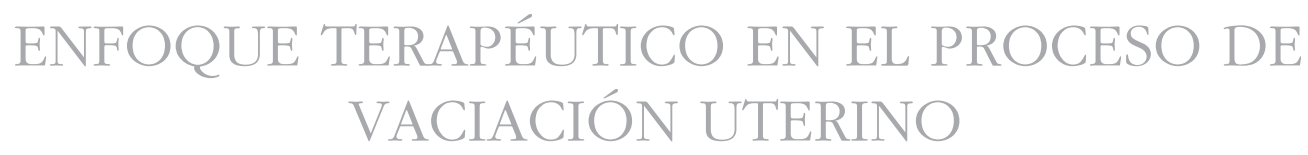

\author{
Cassiana Pinheiro de Araújo ${ }^{1}$ \\ Adélia Cristina Vieira de Rezende Dornelas ${ }^{2}$ \\ António Manuel Sousa ${ }^{3}$
}

\begin{abstract}
Como citar este artigo: Araújo CP, Dornelas ACVR, Sousa AM. Abordagem terapêutica no processo de esvaziamento uterino. Rev baiana enferm. 2018;32:e24857.

Objetivos: identificar a terapêutica utilizada no processo de esvaziamento uterino e traçar o perfil obstétrico das mulheres assistidas nesse processo. Método: pesquisa quantitativa, retrospectiva, de caráter exploratório-descritivo, constituída por 466 prontuários de mulheres internadas para esvaziamento uterino, no período de junho de 2015 a junho de 2016. Resultados: a idade variou entre 14 e 43 anos, o principal diagnóstico foi o aborto incompleto, responsável por $44,85 \%$ das internações, a terapêutica inicial mais utilizada foi o misoprostol (57,71\%) e o tempo de internação até o esvaziamento uterino variou entre 0,20 e 137 horas. Conclusão: o perfil obstétrico da mulher que vivencia a perda gestacional não difere do encontrado nacionalmente; a curetagem foi a terapêutica final mais utilizada acrescida de alguns desfechos desfavoráveis, como maior tempo de internação e exposição excessiva a medicação.
\end{abstract}

Descritores: Saúde da mulher. Abortamento. Métodos terapêuticos. Assistência à saúde.

Objectives: identify the therapeutics used in the uterine evacuation process and outline the obstetric profile of the women attended in this process. Method: quantitative, retrospective, exploratory and descriptive research, consisting of 466 records of women hospitalized for uterine evacuation between June 2015 and June 2016. Results: ages ranged between 14 and 43 years. The main diagnosis was incomplete abortion, responsible for $44.85 \%$ of the hospitalizations. The most used initial therapy was misoprostol (57.71\%) and the length of hospitalization before the uterine evacuation ranged between 0.20 and 137 bours. Conclusion: the obstetric profile of women who experience gestational loss does not differ from the profile found in Brazil; curettage was the most used final therapy, in addition to some unfavorable outcomes, such as longer hospitalization and excessive exposure to medication.

Descriptors: Women's health. Abortion. Therapeutic approaches. Delivery of Health Care.

Objetivos: identificar la terapéutica utilizada en el proceso de vaciamiento uterino y trazar el perfil obstétrico de mujeres asistidas en ese proceso. Método: investigación cuantitativa, retrospectiva, de carácter exploratorio-descriptivo, constituida por 466 prontuarios de mujeres internadas para vaciamiento uterino, de junio de 2015 a junio de 2016. Resultados: edad entre 14 y 43 años, el principal diagnóstico fue el aborto incompleto, responsable por 44,85\% de

Enfermeira. Especialista em Enfermagem Obstétrica. Manaus, Amazonas, Brasil. cassianapinheiro@gmail.com

Enfermeira. Especialista em Enfermagem Obstétrica. Mestranda em Ciências da Saúde pela Universidade de São Paulo. Docente do curso de Enfermagem da Escola Superior da Saúde, Universidade do Estado do Amazonas. Manaus, Amazonas, Brasil.

3 Biólogo. Especialista em Microbiologia. Mestre em Epidemiologia. Doutor em Saúde Coletiva. Professor Adjunto da Escola de Ciências da Saúde, Universidade do Estado do Amazonas. Manaus, Amazonas, Brasil. 
las internaciones, la terapéutica inicial más utilizada fue el misoprostol (57,71\%) y el tiempo de internación hasta el vaciamiento uterino varió entre 0,20 y 137 horas. Conclusión: el perfil obstétrico de la mujer que vive la pérdida gestacional no difiere de lo encontrado nacionalmente; el curetaje fue la terapia final más utilizada, sumada de algunos resultados desfavorables, como mayor tiempo de internación y exposición excesiva a la medicación.

Descriptores: Salud de la mujer. Aborto. Métodos terapéuticos. Prestación de atención de salud.

\section{Introdução}

Do ponto de vista médico, abortamento é conceituado como a interrupção da gravidez até a $20^{\underline{a}}$ ou $22^{\underline{a}}$ semana de gestação e com produto de concepção pesando menos que 500 g. Já o aborto é o produto eliminado no abortamento ${ }^{(1)}$. Em substituição à terminologia médica, a fim de minimizar os estigmas psicológicos, sociais e legais inerentes aos termos "aborto" e "abortamento", o Royal College of Obstetricians and Gynaecologists (RCOG), desde 1997, recomenda o termo perda gestacional, porém, no Brasil, o Ministério da Saúde ainda não reconhece tal termo $^{(2)}$.

A prevalência da perda gestacional varia, mundialmente, entre 15 a 20\% das gestações clinicamente confirmadas, ocorrendo a maior parte antes da $12^{\text {a }}$ semana de gestação ${ }^{(3)}$. É um evento comum entre as mulheres brasileiras. De acordo com a Pesquisa Nacional de Aborto (PNA) realizada em 2016, aproximadamente uma em cada cinco mulheres já vivenciou tal acontecimento. A PNA 2016, assim como a PNA 2010, aponta uma frequência maior entre mulheres mais jovens, com faixa etária que vai dos 18 aos 29 anos $(51,8 \%)$, com a quase metade dessa população (48\%) necessitando de internação para finalizar o abortamento. Considerando que grande parte desses eventos são ilegais e realizados fora das unidades de saúde, a perda gestacional permanece como um dos maiores problemas de saúde pública do Brasil, seja por risco à saúde daquelas que a vivenciam, seja pelos custos do sistema de saúde para prestar assistência ${ }^{(4)}$.

No Brasil, as regiões Norte e Nordeste destacam-se com as maiores taxas de abortamento e os menores índices de redução. A mortalidade materna é apenas uma fração dessa problemática que envolve ainda o déficit na qualidade da assistência à saúde sexual e reprodutiva das mulheres, dificuldade de acesso aos serviços de saúde, baixa escolaridade, baixa renda. Além disso, os dados referentes à hospitalização são fatores que circundam essa temática e contribuem para sua magnitude ${ }^{(5)}$.

Vários aspectos influenciam na tomada de decisão terapêutica da perda gestacional, como idade gestacional definida, complicações presentes, infecções e algumas contraindicações. Por isso, a necessidade de atenção e de prestar assistência de qualidade é imperiosa ${ }^{(2-3)}$.

O tratamento para esvaziamento do útero abrange desde a conduta ativa até a conduta expectante. A primeira inclui os métodos farmacológicos, como misoprostol oral, sublingual ou vaginal associado ou não ao mifepristone por via oral, dependendo do tempo de gestação; e cirúrgicos, como aspiração manual intrauterina (AMIU), a vácuo ou elétrica, para gravidezes de até 12 a 14 semanas de gestação ${ }^{(3-6)}$; e a curetagem uterina, largamente utilizada para esvaziamento uterino em idades gestacionais mais avançadas ${ }^{(7)}$. Este, porém, é considerado um procedimento obsoleto pela Organização Mundial da Saúde (OMS), devendo ser substituído pela aspiração a vácuo ou métodos farmacológicos ${ }^{(6)}$. A conduta expectante, que resulta na completa expulsão do produto da concepção, tem intervalo variado até a expulsão, apresentando o risco de infecção e complicações hemorrágicas como fatores preocupantes ${ }^{(3,7)}$. No entanto, decidir sobre qual a melhor conduta no tratamento da perda gestacional é ainda um grande desafio dos prescritores na atualidade, refletindo a falta de 
informações baseadas em evidências científicas que permitam uma conduta segura e eficaz ${ }^{(3)}$.

Este estudo teve como objetivos identificar a terapêutica utilizada no processo de esvaziamento uterino e traçar o perfil obstétrico das mulheres assistidas nesse processo.

\section{Método}

Estudo quantitativo, retrospectivo, de caráter exploratório-descritivo, realizado em uma Maternidade pública na cidade de Manaus, Amazonas, Brasil. Trata-se de uma das unidades de referência para assistência obstétrica do estado, inclusive para o atendimento de gravidez de alto risco. A pesquisa foi aprovada pelo Comitê de Ética em Pesquisa (CEP) da Universidade do Estado do Amazonas (UEA), CAEE n. 60896616.5.0000.5016, em dezembro de 2016, Parecer n. 1.871.349.

A amostra foi constituída pelos prontuários das pacientes que se internaram na instituição citada, por perda gestacional, no período de junho de 2015 a junho de 2016. Foram utilizados os seguintes critérios de inclusão: idade gestacional até 22 semanas, de acordo com a data da última menstruação (DUM) ou exame de ultrassonografia (USG), diagnóstico de óbito fetal/ embrionário, abortamento incompleto, gravidez molar, abortamento em curso, aborto legal/induzido, gravidez anembrionada e todas as idades. Foram excluídos os prontuários das mulheres que não apresentavam comprovação de idade gestacional, em que o concepto tenha pesado acima de $500 \mathrm{~g}$, prontuários com dados ilegíveis, dados clínicos não compatíveis com diagnóstico, informações conflitantes, pacientes transferidas ou evadidas antes do esvaziamento uterino e as mulheres indígenas, devido à falta de tempo hábil para atender a todo o processo burocrático exigido nas pesquisas realizadas com indígenas.

O quantitativo dos prontuários incluídos na pesquisa baseou-se no número de internações realizadas nos anos de 2015 e 2016 para esvaziamento uterino. Em seguida, os prontuários foram identificados diretamente nos arquivos do Serviço de Arquivo Médico e Estatística (SAME) da instituição, com base no diagnóstico de internação, configurando uma população de 782 prontuários. Posteriormente foram selecionados aqueles que atendiam aos critérios de inclusão e exclusão, totalizando uma amostra de 466 prontuários selecionados, com base no índice de confiança para a amostra de 99,9\%.

A coleta de dados foi feita por meio de um formulário construído pelas pesquisadoras, preenchido com os dados extraídos apenas do prontuário e contendo as seguintes variáveis: dados obstétricos, diagnóstico de internação, conduta terapêutica utilizada, necessidade de tratamento cirúrgico, queixas da paciente, necessidade de medicação, tempo até o esvaziamento uterino, tempo de internação hospitalar, complicações.

A análise dos dados foi realizada por meio da análise descritiva, com o apoio do programa Epi Info versão 7. Os resultados foram apresentados em tabelas de distribuição de frequência.

Em consonância com a Resolução do Conselho Nacional de Saúde n. 466, de 12 de dezembro de $2012^{(8)}$, que dispõe sobre a pesquisa envolvendo seres humanos, foi solicitada a Dispensa do Termo de Consentimento Livre e Esclarecido (TCLE), uma vez que se trata de uma pesquisa retrospectiva com utilização de dados dos prontuários.

\section{Resultados}

Das 466 mulheres incluídas, 191 (40,99\%) mantinham uma união estável, 141 (30,26\%) eram solteiras, 101 (21,67\%) eram casadas e somente $1(0,21 \%)$ era divorciada. Em 32 (6,87\%) prontuários essa informação foi ignorada. Quanto ao local de residência, $447(95,92 \%)$ moravam na cidade de Manaus, 18 (3,87\%) residiam no interior do estado e $1(0,21 \%)$, em outro estado brasileiro.

Das mulheres atendidas 180 (38,63\%) foram internadas com alguma dilatação cervical, 245 (52,58\%) sem dilatação e em 41 (8,80\%) prontuários esse dado não foi informado. Sobre o acompanhamento pré-natal, 296 (63,52\%) não faziam acompanhamento, 63 (13,52\%) já tinham pelo menos uma consulta e em 107 (22,96\%) prontuários essa informação foi ignorada. 
A faixa etária variou entre 14 e 43 anos, com média de idade de 26,8 anos ( $\mathrm{DP}=7,35)$. Em relação aos dados obstétricos 370 (79,40\%) apresentavam idade gestacional menor que 13 semanas de gestação, com uma média de idade gestacional de 10,2 semanas ( $\mathrm{DP}=3,63), 338$ (72,53\%) não tinham nenhum aborto anterior e 128 (27,47\%) mulheres já haviam tido algum aborto. Não foi investigado se esses abortamentos foram provocados ou espontâneos. O número de gestações variou entre 1 e 13; 214 $(45,93 \%)$ mulheres atendidas estavam entre a segunda e terceira gestação, tinham entre 1 e 8 partos vaginais e de 1 a 3 cesarianas (Tabela 1 ).

Tabela 1 - Distribuição das mulheres atendidas em processo de esvaziamento uterino segundo a faixa etária, idade gestacional, número de gestações, parto, aborto e tipo de parto. Manaus, Amazonas, Brasil - 2015-2016 ( $\mathrm{N}=466)$

\begin{tabular}{|c|c|c|c|c|}
\hline \multirow{2}{*}{ Variável } & \multicolumn{2}{|c|}{ Frequência } & \multirow{2}{*}{ Média } & \multirow{2}{*}{ Desvio Padrão } \\
\hline & $\mathbf{n}$ & $\%$ & & \\
\hline Faixa etária & 466 & 100,00 & & \\
\hline $14-16$ & 24 & 5,15 & 26,75 & 7,35 \\
\hline $17-19$ & 71 & 15,24 & & \\
\hline $20-22$ & 65 & 13,95 & & \\
\hline $23-25$ & 69 & 14,81 & & \\
\hline $26-29$ & 74 & 15,88 & & \\
\hline $30-34$ & 72 & 15,45 & & \\
\hline $35-39$ & 62 & 13,30 & & \\
\hline$\geq 40$ & 29 & 6,22 & & \\
\hline Idade gestacional & 466 & 100,00 & & \\
\hline$\leq 12,6$ semanas & 370 & 79,40 & 10,20 & 3,63 \\
\hline 13 a 22 semanas & 96 & 20,60 & & \\
\hline Número de abortos & 466 & 100,00 & & \\
\hline 0 & 338 & 72,53 & 0,36 & 0,68 \\
\hline $1-2$ & 121 & 25,97 & & \\
\hline$\geq 3$ & 7 & 1,49 & & \\
\hline Número de gestações & 466 & 100,00 & & \\
\hline 1 & 114 & 24,46 & 2,93 & 1,91 \\
\hline $2-3$ & 214 & 45,93 & & \\
\hline$\geq 4$ & 138 & 29,61 & & \\
\hline Número de partos vaginais & 466 & 100,00 & & \\
\hline 0 & 211 & 45,28 & & \\
\hline 1 & 103 & 22,10 & 1,22 & 1,54 \\
\hline $2-3$ & 114 & 24,47 & & \\
\hline$\geq 4$ & 38 & 8,15 & & \\
\hline Número de partos cesáreos & 466 & 100,00 & & \\
\hline 0 & 346 & 74,25 & 0,35 & 0,65 \\
\hline$\geq 1$ & 120 & 25,75 & & \\
\hline
\end{tabular}

Fonte: Elaboração própria.

Dentre os diagnósticos para internação, o aborto incompleto foi o principal, sendo responsável por 209 (44,85\%) internações para esvaziamento uterino, seguido do óbito fetal, com 163 $(34,98 \%)$. A gravidez molar e o aborto infectado foram os motivos menos citados, sendo cada um responsável, respectivamente, por $3(0,64 \%)$ e $4(0,86 \%)$ internações. Sobre a terapêutica de escolha, o misoprostol foi o mais utilizado (59,01\%) seguido do procedimento de curetagem (37,55\%), ambos utilizados associados ou não. A conduta expectante foi indicada a 38 $(8,15 \%)$ mulheres. Vale ressaltar que foi considerada como conduta expectante o método sem 
intervenção farmacológica ou cirúrgica para o esvaziamento uterino, com as mulheres submetidas a esse método permanecendo internadas na instituição (Tabela 2).

Tabela 2 - Distribuição do motivo da internação hospitalar e terapêutica utilizada para esvaziamento uterino. Manaus, Amazonas, Brasil - 2015-2016 ( $\mathrm{N}=466)$

\begin{tabular}{l|cc}
\hline \multirow{2}{*}{ Variável } & \multicolumn{2}{c}{ Frequência } \\
\cline { 2 - 3 } Motivo da internação & $\mathbf{n}$ & $\mathbf{4 6}$ \\
Abortamento em curso & 52 & $\mathbf{1 0 0 , 0 0}$ \\
Aborto incompleto & 209 & 11,16 \\
Aborto infectado & 4 & 44,85 \\
Gravidez anembrionada & 35 & 0,86 \\
Gravidez Molar & 3 & 7,51 \\
Óbito fetal/ embrionário/ aborto retido & 163 & 0,64 \\
Indicação terapêutica & $\mathbf{5 2 3}$ & 34,98 \\
Aspiração Manual Intrauterina & $35^{*}$ & 7,51 \\
Curetagem & $175^{*}$ & 37,55 \\
Misoprostol & $275^{*}$ & 59,01 \\
Conduta expectante & $38^{*}$ & 8,15 \\
Misoprostol associado ao tratamento cirúrgico & $\mathbf{2 7 5}$ & $\mathbf{1 0 0 , 0 0}$ \\
Não & 13 & 4,73 \\
Aspiração Manual Intrauterina & 48 & 17,45 \\
Curetagem & 213 & 77,46 \\
Aspiração Manual Intrauterina + curetagem & 1 & 0,36 \\
Conduta expectante associada a outro método & $\mathbf{3 8}$ & $\mathbf{1 0 0 , 0 0}$ \\
terapêutico & & 31,58 \\
Não & 12 & 2,63 \\
Aspiração Manual Intrauterina & 1 & 52,63 \\
Curetagem & 20 & 13,16 \\
Misoprostol & 5 & \\
\hline
\end{tabular}

Fonte: Elaboração própria.

* Métodos terapêuticos associados.

Como terapia farmacológica foi utilizado somente o misoprostol, sendo encontrados 19 esquemas terapêuticos diferentes prescritos, variando de 1 a 5 doses administradas, com média de 1,35 ( $\mathrm{DP}=0,93$; $\mathrm{CV}=68.89 \%)$ dose, independentemente do esquema terapêutico utilizado. Das mulheres submetidas a essa terapêutica, 212 (76,26\%) utilizaram entre 1 e 2 doses, 30 $(10,79 \%)$ com 3 ou mais doses administradas. Das doses prescritas, $12,95 \%$ não foram administradas e pelo menos 3 mulheres utilizaram mais de um esquema farmacológico.

Quanto à necessidade de tratamento cirúrgico associado à terapêutica inicial, 262 (95,27\%) mulheres que utilizaram a terapia medicamentosa e 26 (68,42\%) daquelas que iniciaram com a conduta expectante necessitaram de algum tipo de tratamento cirúrgico.

O tempo de internação até o esvaziamento uterino variou entre 0,20 min e 137 horas. Já a internação até a alta hospitalar variou entre 4,30 a 220 horas.

Dentre as queixas apresentadas, o sangramento moderado foi citado por 215 (46,14\%) mulheres, $176(37,77 \%)$ referiram dor e 175 (37,55\%) relataram sangramento discreto, 20 (4,29\%) apresentaram hemorragia, $4(0,86 \%)$ apresentaram vômito, febre foi citada por $2(0,43 \%)$ mulheres, mal-estar, hipotensão, calafrio, diarreia, fraqueza cada um foi citado apenas uma vez, e 42 (9,01\%) não apresentaram nenhuma queixa. 
Tabela 3 - Distribuição da média de tempo para esvaziamento uterino e internação de acordo com a terapêutica utilizada. Manaus, Amazonas, Brasil - 2015-2016 ( N = 466)

\begin{tabular}{|c|c|c|c|c|c|c|c|c|}
\hline \multirow{2}{*}{ Variável } & \multicolumn{3}{|c|}{$\begin{array}{c}\text { Tempo (h) esvaziamento } \\
\text { uterino }\end{array}$} & \multirow{2}{*}{$\mathbf{C V}$} & \multicolumn{3}{|c|}{ Tempo (h) internação } & \multirow{2}{*}{$\mathbf{C V}$} \\
\hline & Média & $\begin{array}{l}\text { Mínimo- } \\
\text { máximo }\end{array}$ & $\begin{array}{l}\text { Desvio } \\
\text { Padrão }\end{array}$ & & Média & $\begin{array}{l}\text { Mínimo- } \\
\text { máximo }\end{array}$ & $\begin{array}{l}\text { Desvio } \\
\text { Padrão }\end{array}$ & \\
\hline $\begin{array}{l}\text { Aspiração Manual } \\
\text { Intrauterina }\end{array}$ & 21,53 & $1,00-75,00$ & 5,79 & 27,14 & 43,28 & $4,30-96,00$ & 6,73 & 15,55 \\
\hline Curetagem & 15,55 & $0,20-86,00$ & 8,07 & 51,90 & 44,32 & $6,4-201,25$ & 16,09 & 36,64 \\
\hline Misoprostol & 36,13 & $2,00-137,00$ & 16,52 & 45,72 & 63,30 & $16,15-220,00$ & 21,11 & 33,56 \\
\hline Conduta expectante & 28,22 & $0,20-91,00$ & 6,71 & 24,12 & 67,02 & $13,00-211,37$ & 12,85 & 19,29 \\
\hline
\end{tabular}

Fonte: Elaboração própria.

CV = Coeficiente de variação.

Em relação à necessidade de medicação de acordo com a terapêutica utilizada, como mostra a Tabela 4, o analgésicos foi utilizado por 19 (54,29\%) pacientes que se submeteram ao método de aspiração manual intrauterina (AMIU), 69 (39,43\%) para curetagem, $114(41,45 \%)$ para o misoprostol e $20(52,63 \%)$ para a conduta expectante. A necessidade de antibióticos foi observada em 4 (11,43\%) pacientes para o AMIU, 37 $(21,14 \%)$ para a curetagem, $41(14,91 \%)$ para o misoprostol e $9(23,68 \%)$ para a conduta expectante. O uterotônico foi utilizado por $15(42,86 \%)$ pacientes que usaram o AMIU, 130 (74,29\%) das que realizaram a curetagem, $182(66,18 \%)$ para o misoprostol e $28(73,68)$ para a conduta expectante. Utilizaram hemoderivados, 7 (4,00\%) pacientes que realizaram a curetagem, 5 (1,82\%) que utilizaram o misoprostol e $3(7,89 \%)$ que iniciaram com a conduta expectante; para aquelas que realizaram o AMIU, não foi necessário.

Tabela 4 - Distribuição da necessidade de medicamentos utilizados de acordo com a indicação terapêutica. Manaus, Amazonas, Brasil - 2015-2016 ( $\mathrm{N}=466)$

\begin{tabular}{l|c|c|c|c|c|c|c|c}
\hline \multirow{2}{*}{ Terapêutica } & \multicolumn{7}{c|}{ Analgésico } & \multicolumn{7}{c|}{ Antibiótico } & \multicolumn{2}{c}{ Uterotônico } & \multicolumn{2}{c}{ Hemoderivado } \\
\cline { 2 - 9 } & \multicolumn{8}{c}{ Necessidade de medicação } \\
\cline { 2 - 9 } & $\mathbf{n}$ & $\mathbf{0}$ & $\mathbf{n}$ & $\mathbf{\%}$ & $\mathbf{n}$ & $\mathbf{\%}$ & $\mathbf{n}$ & $\mathbf{\%}$ \\
\hline Conduta expectante & 20 & 52,63 & 9 & 23,68 & 28 & 73,68 & 3 & 7,89 \\
Aspiração Manual Intrauterina & 19 & 54,29 & 4 & 11,43 & 15 & 42,86 & 0 & 0,00 \\
Curetagem & 69 & 39,43 & 37 & 21,14 & 130 & 74,29 & 7 & 4,00 \\
Misoprostol & 114 & 41,45 & 41 & 14,91 & 182 & 66,18 & 5 & 1,82 \\
\hline
\end{tabular}

Fonte: Elaboração própria.

A correlação entre os tipos de tratamento oferecidos e a ocorrência de complicações (Tabela 5) mostrou que todos eles apresentaram a ocorrência, de forma associada ou não. As complicações encontradas foram: sangramento persistente, hemorragia, endometrite, restos ovulares, hematoma de colo uterino e perfuração uterina, totalizando $8(1,72 \%)$ casos. O procedimento de misoprostol foi o que mais apresentou complicações, com 5 casos. Das 8 ocorrências de complicações, quatro mulheres precisaram ser submetidas a um novo procedimento cirúrgico, dentre eles: laparotomia exploradora (2 casos) e curetagem (2 casos). 
Tabela 5 - Distribuição da frequência das indicações terapêuticas de acordo com as complicações apresentadas. Manaus, Amazonas, Brasil - 2015-2016 ( $\mathrm{N}=466)$

\begin{tabular}{|c|c|c|c|c|c|}
\hline \multirow{3}{*}{ Indicação terapêutica } & \multicolumn{5}{|c|}{ Complicação após a terapêutica utilizada } \\
\hline & \multicolumn{2}{|c|}{ Não } & \multicolumn{2}{|c|}{ Sim } & \multirow{2}{*}{ Total } \\
\hline & $\mathbf{n}$ & $\%$ & $\mathbf{n}$ & $\%$ & \\
\hline Total & 513 & & 8/10 & & 523 \\
\hline Aspiração Manual Intrauterina & 34 & $97,14 \%$ & 1 & $2,86 \%$ & 35 \\
\hline Curetagem & 172 & $98,29 \%$ & $3^{*}$ & $1,71 \%$ & 175 \\
\hline Misoprostol & 270 & $98,18 \%$ & $5^{*}$ & $1,82 \%$ & 275 \\
\hline Conduta expectante & 37 & $97,37 \%$ & $1 *$ & $2,63 \%$ & 38 \\
\hline
\end{tabular}

Fonte: Elaboração própria.

* Métodos terapêuticos associados.

\section{Discussão}

A maternidade em estudo, no ano de 2015, teve um total de 665 internações para esvaziamento uterino. Em 2016, esse número chegou a 526 internações, tendo um declínio de 20,90\% desse atendimento em relação ao ano anterior, com o abortamento representando $7 \%$ das internações totais da instituição. O esvaziamento uterino pós-abortamento, em 2015, passou a ser o segundo em procedimentos cirúrgicos obstétricos em âmbito nacional. No Amazonas, este é o terceiro procedimento mais realizado nas maternidades públicas do estado, de acordo com o Sistema de Internação Hospitalar do SUS $(\mathrm{SIH} / \mathrm{SUS})^{(9)}$.

De acordo com a última Pesquisa Nacional de Aborto, realizada em 2016 no Brasil, por meio de inquérito domiciliar de mulheres alfabetizadas com idade entre 18 e 49 anos, que entrevistou 2.002 voluntárias, 251 (13\%) já haviam realizado aborto em alguma etapa de sua vida, 102 (40,64\%) tinham entre 20 e 29 anos - caracterizando-se por uma frequência maior entre as mulheres mais jovens e declinando nas mulheres mais velhas -, 163 (60\%) eram casadas ou viviam em união estável ${ }^{(4)}$.

Em pesquisa realizada em Recife (PE), no Centro de Atenção à Mulher (CAM) do Instituto de Medicina Integral Professor Fernando Figueira (IMIP), no período de 2005-2006, com 160 mulheres nessa mesma faixa etária, no intuído de investigar as características sociodemográficas e reprodutivas das mulheres hospitalizadas por abortamento, $78(48,9 \%)$ das participantes tinham entre 20 e 30 anos, 144 (90,1\%) tinham companheiro, $89(55,7 \%)$ tinham pelo menos um filho, 116 (72,5\%) não tinham abortos anteriores, 89 (55,7\%) apresentaram abortamento precoce e 9 (5,6\%) não tinham idade gestacional conhecida $^{(10)}$.

O perfil obstétrico das mulheres atendidas na instituição pesquisada não se distinguiu do perfil nacional das mulheres que já vivenciaram o aborto, tendo a maior incidência ocorrido no período considerado de maior atividade reprodutiva, que vai dos 20 aos 30 anos de idade. Um fato que chamou a atenção foi a porcentagem significativa de dados obstétricos e de avaliação clínica ignorados. Esta ausência acaba dificultando a caracterização do cenário real e até a avaliação da conduta tomada pelas mulheres que vivenciaram a perda gestacional.

Um ensaio clínico realizado na Santa Casa de Misericórdia de Sobral (CE), em 2006, com 41 pacientes com gestação interrompida entre $7 \mathrm{e}$ 12 semanas de gestação, avaliou a eficácia do uso de misoprostol via vaginal em substituição à curetagem uterina, utilizando uma única dose de $800 \mathrm{mcg}$. Esse procedimento alcançou uma taxa de sucesso de 80,5\%, com uma média de tempo até o esvaziamento de aproximadamente 13 horas, e foi mais expressivo quando utilizado na idade gestacional abaixo de 8 semanas (96,2\%). Historicamente, acreditava-se que todas as perdas gestacionais deveriam ser 
consideradas incompletas, com a necessidade de procedimento cirúrgico para retirada de tecidos placentários, a fim de evitar complicações ${ }^{(3)}$. No entanto, os resultados do ensaio sugeriram que o misoprostol, comparado com outros análogos de prostaglandinas, na dose de $800 \mathrm{mcg}$ via vaginal, era uma alternativa segura e eficaz em relação à curetagem uterina ${ }^{(7)}$.

A presente pesquisa vai de encontro aos demais estudos, ao apresentar uma taxa de sucesso de apenas 4,69\% para o uso do misoprostol, apesar de o estudo comparado ser realizado com mulheres em idade gestacional até 12 semanas. Isso pode estar relacionado aos diferentes esquemas terapêuticos utilizados, apesar de existirem protocolos específicos lançados pelo Ministério da Saúde. Outro fator a ser considerado é a superlotação na instituição de saúde, o número de doses (12,95\%) não administradas e a falta de leitos para administração da medicação, relatada nos prontuários. Pôde-se concluir, analisando o sucesso do tratamento farmacológico, que é de grande relevância a construção de condutas padronizadas visando a eficácia do tratamento, diminuindo, consequentemente, os riscos de complicações ${ }^{(3)}$.

Um estudo analítico-descritivo realizado no Centro Obstétrico do Hospital Universitário de Santa Maria (RS), com 30 gestantes de até 12 semanas de gestação e diagnóstico de abortamento incompleto, comparou eficácia, complicação, perdas sanguíneas e tempo de internação entre os procedimentos de curetagem e AMIU. O tempo até o esvaziamento foi em média de 12,4 horas para curetagem e 8,0 horas para o AMIU, sem diferença significativa entre o tempo de realização do procedimento cirúrgico. Em tal estudo, o tempo de permanência hospitalar das pacientes submetidas à curetagem foi, em média, de 18 horas e o grupo que realizou AMIU foi de 10,7 horas. Ambos os grupos tiveram igual eficácia, sem necessidade de novo procedimento cirúrgico. Apesar de não se ter encontrado diferença com significância estatística para a perda sanguínea entre os dois grupos, as mulheres submetidas ao AMIU apresentaram menor variação dos parâmetros hematimétricos ${ }^{(11)}$.
A curetagem uterina foi o segundo tratamento mais observado, responsável por 175 (37,55\%) indicações dessa terapêutica. O número de mulheres que utilizou esse tratamento alcançou o total de 409 (87,77\%), quando avaliado o procedimento final para o esvaziamento, sendo este associado à conduta expectante e ao misoprostol, alcançando eficácia desse método de $99,27 \%$. Foram encontrados 3 casos $(0,73 \%)$ de complicações para esse método, com 1 (0,25\%) paciente apresentando necessidade de novo tratamento cirúrgico (laparotomia exploradora) por perfuração uterina.

A AMIU foi responsável por 85 (18,24\%) indicações para o esvaziamento uterino, apesar de $370(79,40 \%)$ pacientes terem apresentado abortamento precoce. De acordo com a literatura, é indicada em gestações com menos de 12 semanas em função do tamanho do útero ${ }^{(2)}$. O tempo médio até o esvaziamento uterino foi superior ao tempo encontrado para a curetagem, no entanto o tempo médio de internação até a alta hospitalar foi de aproximadamente 43 horas, sendo menor tempo de internação entre as terapêuticas utilizadas, menor necessidade de uterotônicos, antibióticos e hemoderivados.

A eficácia do tratamento cirúrgico para o esvaziamento uterino mantém-se com maior efetividade em relação aos outros métodos utilizados para esvaziamento uterino na perda gestacional, porém sua utilização não é isenta de $\operatorname{riscos}^{(3)}$. De acordo com a literatura que compara os tratamentos cirúrgicos para a perda gestacional, o AMIU apresenta vantagem em relação à curetagem: menor tempo de internação, o que contribui para redução dos custos hospitalares; substituição na anestesia geral ou bloqueio raquidiano por analgesia, podendo ser associada ou não ao bloqueio paracervical; não requer a presença do anestesiologista e está associada ao baixo risco de complicações, como perfuração uterina, laceração de colo e hemorragia genital. Esta é também a técnica de eleição para o aborto infectado, sendo recomendado pela OMS e pela Federação Internacional de Ginecologia e Obstetrícia (FIGO). Uma revisão da Biblioteca Cochrane revela que a aspiração é um método 
mais rápido, menos doloroso, com menor perda sanguínea e mais seguro que a curetagem ${ }^{(12)}$. Importante lembrar que a OMS considera a curetagem uterina um método obsoleto para o esvaziamento uterino, sendo recomendada a utilização da aspiração a vácuo ${ }^{(6)}$.

Mesmo com a disponibilidade do método de aspiração intrauterina (AMIU), a curetagem ainda é predominante, não só no presente estudo, mas no âmbito nacional. A escolha desse método pode estar relacionada ao alto custo do vácuo aspirador e a necessidade de profissionais capacitados para tal procedimento ${ }^{(3)}$, o que pode estar refletindo também no tempo de internação até o esvaziamento uterino das mulheres que foram submetidas ao AMIU na atual pesquisa, que foi de 43,28 horas, além de expô-las a maiores riscos de infecção, pela permanência no ambiente hospitalar, e dos custos pelo próprio tempo de internação. Ainda assim, a presente pesquisa aponta o AMIU como um método seguro, com menor variação de tempo entre os métodos ativos (cirúrgico e farmacológico) até o esvaziamento uterino e também no tempo de internação hospitalar, entre todos os métodos disponíveis na instituição.

A conduta expectante foi o método terapêutico menos utilizado (8,15\%), sendo, geralmente, associada inicialmente ao diagnóstico de abortamento em curso. Entretanto, 26 (68,42\%) pacientes submetidas a esse método necessitaram de algum tratamento associado para o esvaziamento uterino. As mulheres que utilizaram essa terapêutica foram as que mais necessitaram de uterotônicos, antibióticos e hemoderivados. Em relação à ocorrência de complicação, foi encontrado um caso $(2,63 \%)$ que exigiu procedimento cirúrgico para correção.

Quando comparada a conduta expectante ao tratamento cirúrgico e farmacológico, essa chegou a apresentar uma eficácia de, em média, 73\% como terapêutica para a perda gestacional. Quanto à ocorrência de sangramento e complicações, apresenta os maiores índices em relação ao tratamento cirúrgico, quando comparados níveis de hemoglobina, quantidade de sangramento e necessidade de transfusão. Quanto à infecção pélvica, não se evidencia diferença entre os métodos disponíveis ${ }^{(3)}$.

Apesar de menores taxas sucesso, o presente estudo corrobora dados da literatura em relação às queixas e necessidade de medicação e hemoderivados e de tratamento associado. O sucesso da conduta expectante como método terapêutico depende de uma avaliação criteriosa do tempo de gestação, tipo de perda, tempo de seguimento e características do material encontrado. Por esta razão, a eficiência desse método para o esvaziamento uterino pode chegar a 90\% ${ }^{(3)}$.

Não foram encontrados métodos terapêuticos alternativos para o tratamento no esvaziamento uterino na instituição pesquisada, como, por exemplo, a acupuntura, apesar da existência de estudos internacionais que apontam esse método como alternativa eficaz. Também não foi encontrado nenhum estudo disponível, no âmbito nacional, sobre a utilização da acupuntura para esvaziamento uterino, evidenciando que esta prática ainda não é disponível e acessível nas instituições públicas de saúde que atendem à mulher na perda gestacional ${ }^{(5)}$.

As principais limitações do estudo resultaram do preenchimento incompleto do prontuário das participantes, no que se refere à idade, idade gestacional definida, diagnóstico e terapêutica utilizada, falta de legitimidade na escrita, procedimentos realizados e complicações apresentadas, o que resultou em uma amostra inferior à estimada inicialmente, resultando em dados ainda inferiores ao exato cenário da mulher que vivencia a perda gestacional.

\section{Conclusão}

O perfil da mulher atendida no serviço de saúde pública amazonense, com diagnóstico de perda gestacional, não difere do encontrado nacionalmente, sendo a principal faixa etária acometida entre 20 e 30 anos de idade e apresentando mais de uma gestação.

Apesar da ocorrência de perda gestacional precoce, a conduta corrobora a aplicada ainda em outras regiões do país, sendo a curetagem o principal método terapêutico utilizado para 
o esvaziamento uterino. Observaram-se alguns desfechos desfavoráveis, como o tempo maior de internação e exposição excessiva à medicação. Esses podem estar relacionados a fatores logísticos, orçamentários e de qualificação profissional e não somente à terapêutica utilizada.

Pôde-se concluir que o perfil obstétrico da mulher que vivencia a perda gestacional não difere do encontrado nacionalmente; a curetagem foi a terapêutica final mais utilizada, acrescida de alguns desfechos desfavoráveis, como maior tempo de internação e exposição excessiva a medicação.

A superlotação e a falta de padronização da assistência fornecida, por exemplo, refletem no processo terapêutico de forma negativa, o que sugere a necessidade de mais pesquisas que possam confirmar tais associações.

Com isso, pode-se questionar se a terapêutica utilizada para perda gestacional nos serviços públicos de saúde oferta uma assistência completa e de qualidade à população feminina e se o uso de métodos terapêuticos alternativos não poderia ser adotado com o objetivo de minimizar o tempo de internação e o uso excessivo de medicações, amenizando o sofrimento da mulher submetida ao processo terapêutico do esvaziamento uterino.

\section{Colaborações:}

1. concepção, projeto, análise e interpretação dos dados: Cassiana Pinheiro de Araújo, Adélia Cristina Vieira de Rezende Dornelas e António Manuel Sousa;

2. redação do artigo e revisão crítica relevante do conteúdo intelectual: Cassiana Pinheiro de Araújo, Adélia Cristina Vieira de Rezende Dornelas e António Manuel Sousa;

3. aprovação final da versão a ser publicada: Cassiana Pinheiro de Araújo, Adélia Cristina Vieira de Rezende Dornelas e António Manuel Sousa.

\section{Referências}

1. Brasil. Ministério da Saúde. Manual Técnico de Gestação de Alto Risco. Brasília; 2012.
2. Brasil. Ministério da Saúde. Atenção humanizada ao abortamento: norma técnica. 2a ed. Brasilia; 2011.

3. Camayo FJA, Martins LAB, Cavalli RDC. Perda gestacional retida: tratamento baseado em evidência. FEMINA. 2011;39(1):49-56.

4. Diniz D, Medeiros M, Madeiro A. Pesquisa Nacional de Aborto 2016. Ciênc saúde coletiva [Internet]. 2017 Feb [cited 2017 Apr 10];22(2):653-60. Available from: http://dx.doi. org/10.1590/1413-81232017222.23812016

5. Naguindás P. Papel da acupuntura na indução do trabalho de parto [dissertação]. Covilhã (PT): Universidade da Beira Interior; 2012.

6. Organização Mundial da Saúde. Abortamento seguro: orientação técnica e de políticas para sistemas de saúde. 2a edição. Genebra; 2013.

7. Arcanjo FCN, Ribeiro AS, Teles TG, Macena RHM, Carvalho FHC. Uso do misoprostol em substituição à curetagem uterina em gestações interrompidas precocemente. Rev Bras Ginecol Obstet [Internet]. 2011 June [cited 2017 Apr 12];33(6):276-80. Available from: http://dx.doi. org/10.1590/S0100-72032011000600003

8. Brasil. Ministério da Saúde. Conselho Nacional de Saúde. Resolução n. 466, de 12 de dezembro de 2012. Aprova as diretrizes e normas regulamentadoras de pesquisas envolvendo seres humanos [Internet]. Brasília; 2012 [cited 2017 Apr 18]. Available from: http://bvsms.saude.gov.br/ bvs/saudelegis/cns/2013/res0466_12_12_2012. html

9. Brasil. Ministério da Saúde. Procedimentos hospitalares do SUS por local de internação Amazonas. Sistema de Informações Hospitalares do SUS (SIH/SUS). Brasília; 2015.

10. Ramos KS, Ferreira ALCG, Souza AI. Mulheres hospitalizadas por abortamento em uma Maternidade Escola na cidade do Recife, Brasil. Rev esc enferm USP [Internet]. 2010 Sept [cited 2017 Apr 12];44(3):605-10. Available from: http:// dx.doi.org/10.1590/S0080-62342010000300008

11. Saciloto MP, Konopka CK, Velho MTC, Jobim FC, Resener EV, Muradás RR, et al. Aspiração manual intrauterina no tratamento do abortamento incompleto até 12 semanas gestacionais: uma alternativa à curetagem uterina. Rev Bras Ginecol Obstet [Internet]. 2011 out [cited 2017 Apr 10];33(10):292-6. Available from: http://dx.doi. org/10.1590/S0100-72032011001000004 
12. Nanda K, Peloggia A, Grimes D, Lopez L, Nanda G. Expectant care versus surgical treatment for miscarriage. Cochrane Database Syst Rev (Online). 2012 Mar 14 [cited 2017 Apr 18];(3):CD003518. Available from: doi: 10.1002/14651858.CD003518. pub3
Recebido: 30 de novembro de 2017

Aprovado: 3 de abril de 2018

Publicado: 27 de junho de 2018

A Revista Baiana de Enfermagem utiliza a Licença Creative Commons - Atribuição-NãoComercial 4.0 Internacional. https://creativecommons.org/licenses/by-nc/4.0/ Este artigo é de acesso aberto distribuído sob os termos da Licença Creative Commons (CC BY-NC). Esta licença permite que outros remixem, adaptem e criem a partir do seu trabalho para fins não comerciais e, embora, os novos trabalhos tenham de lhe atribuir o devido crédito e não possam ser usados para fins comerciais, os usuários não têm de licenciar esses trabalhos derivados sob os mesmos termos. 